University of Nebraska - Lincoln

DigitalCommons@University of Nebraska - Lincoln

USDA Wildlife Services - Staff Publications

U.S. Department of Agriculture: Animal and Plant Health Inspection Service

2018

Detection of Inflammation via Volatile Cues in Human Urine

Amy R. Gordon

Karolinska Institute

Bruce A. Kimball

Monell Chemical Senses Center

Kimmo Sorjonen

Karolinska Institute

Bianka Karshikoff

Karolinska Institute

John Axelsson

Karolinska Institute

See next page for additional authors

Follow this and additional works at: https://digitalcommons.unl.edu/icwdm_usdanwrc

Part of the Life Sciences Commons

Gordon, Amy R.; Kimball, Bruce A.; Sorjonen, Kimmo; Karshikoff, Bianka; Axelsson, John; Lekander, Mats; Lundstrom, Johan N.; and Olsson, Mats J., "Detection of Inflammation via Volatile Cues in Human Urine" (2018). USDA Wildlife Services - Staff Publications. 2200.

https://digitalcommons.unl.edu/icwdm_usdanwrc/2200

This Article is brought to you for free and open access by the U.S. Department of Agriculture: Animal and Plant Health Inspection Service at DigitalCommons@University of Nebraska - Lincoln. It has been accepted for inclusion in USDA Wildlife Services - Staff Publications by an authorized administrator of DigitalCommons@University of Nebraska - Lincoln. 


\section{Authors}

Amy R. Gordon, Bruce A. Kimball, Kimmo Sorjonen, Bianka Karshikoff, John Axelsson, Mats Lekander, Johan N. Lundstrom, and Mats J. Olsson 
Original Article

\title{
Detection of Inflammation via Volatile Cues in Human Urine
}

\author{
Amy R. Gordon ${ }^{1,2}$, Bruce A. Kimball ${ }^{2,3}$, Kimmo Sorjonen ${ }^{1}$, \\ Bianka Karshikoff ${ }^{1,4-6}$, John Axelsson ${ }^{1,4,5}$, Mats Lekander ${ }^{1,4,5}$, \\ Johan N. Lundström ${ }^{1,2,7, \oplus}$ and Mats J. Olsson ${ }^{1}$
}

'Department of Clinical Neuroscience, Karolinska Institutet, Stockholm, Sweden, ${ }^{2}$ Monell Chemical Senses Center, Philadelphia, PA, USA, ${ }^{U}$ USDA-APHIS-WS, National Wildlife Research Center, Monell Chemical Senses Center, Philadelphia, PA, USA, ${ }^{4}$ Stress Research Institute, Stockholm University, Stockholm, Sweden, ${ }^{5}$ Osher Center for Integrative Medicine, Karolinska Institute, Stockholm, Sweden, ${ }^{6}$ Department of Anesthesiology, Perioperative and Pain Medicine, Stanford University, Palo Alto, CA, USA and 'Department of Psychology, University of Pennsylvania, Philadelphia, PA, USA

Correspondence to be sent to: Mats J. Olsson, Department of Clinical Neuroscience, Karolinska Institutet, Nobels väg 9, Stockholm, Sweden. e-mail: mats.j.olsson@ki.se

Editorial Decision 6 July 2018.

\begin{abstract}
Contagious disease is a major threat to survival, and the cost of relying on the immune system to defeat pathogens is high; therefore, behavioral avoidance of contagious individuals is arguably an adaptive strategy. Animal findings demonstrate the ability to detect and avoid sick individuals by the aid of olfactory cues, and a recent study indicated that human axillary odor also becomes more aversive as a function of immune activation. By injecting healthy human participants with lipopolysaccharide $(0.6 \mathrm{ng} / \mathrm{kg}$ body weight) to experimentally induce inflammation, this study demonstrates that natural daily rhythms of urine odor-its perceived dimensions and volatile profile-are altered within hours of inflammation onset. Whereas healthy human urine decreases in averseness over the course of a single day, inflammation interrupts this process and results in an increased urine odor averseness and an altered volatile composition. These results support the notion that subtle and early cues of sickness may be detected and avoided, thereby complementing the immune system in its role of keeping us alive and healthy.
\end{abstract}

Key words: behavioral immune system, human, inflammation, odor, sickness, urine

\section{Introduction}

Throughout evolution, one of the more common causes of death has been infections (Jones et al. 2012). The threat posed by infection for living organisms is reflected in the fact that genes related to immunity are evolutionary hotspots, with pathogens as a leading cause of change in humans (Fumagalli et al. 2011). Recent evidence suggests that selective pressures have also shaped a behavioral defense against disease (Schaller and Duncan 2007). Indeed, across phyla, behavior adjustment would represent a first line of defense against infection, preventing or reducing parasite load via the detection of sickness cues and the initiation of avoidance behaviors (Curtis 2014), as well as sickness behaviors, to increase recuperation once infected (Dantzer et al. 2008).

It is becoming increasingly clear that most mammals-including humans-rely on olfaction for communication (Wyatt 2014). Among the cues communicated by endogenous volatile compounds are an individual's age (Osada et al. 2003; Mitro et al. 2012), genetic composition (Eggert et al. 1998), kin status (Lundstrom et al. 
2009), and emotional state (de Groot et al. 2012). In addition, a number of nonhuman animal studies have demonstrated that urine from immune-activated individuals contains volatile sickness cues and that these cues initiate an avoidance response in conspecifics (Kavaliers and Colwell 1995a, 1995b; Kiesecker et al. 1999; Ehman and Scott 2001, 2002; Arakawa et al. 2011; Kimball et al. 2014). The variety of immune challenges used in these studies, from parasites to viruses, including vaccines, suggests that the mechanisms regulating the release of such communicative volatile metabolites are not necessarily disease-specific. Furthermore, although these volatile cues could be generated at any stage of the innate or adaptive immune responses, their rapid availability post-administration, within hours of pathogen exposure, appears to exclude adaptive immunity. The quick-acting, general, innate immune response, therefore, is a strong contender for regulating such cues (Kimball et al. 2014).

A common experimental model of the innate immune system's acute inflammatory response utilizes injection of the bacterial endotoxin lipopolysaccharide (LPS), a potent, nonspecific activator of the innate immune system (Dantzer et al. 2008; Beutler 2009). Toll-like receptor 4 (TLR4), expressed on sentinel cells, recognizes and binds LPS, resulting in the production of interleukin-1 $\beta$ (IL-1 $\beta$ ) pro-inflammatory cytokines. Thereafter, IL-1 $\beta$ triggers its own synthesis as well as that of additional, mainly pro-inflammatory cytokines, tumor necrosis factor- $\alpha$ (TNF- $\alpha$ ) and interleukin-6 (IL-6), and anti-inflammatory cytokines, such as interleukin-10 (IL-10) (Karshikoff 2015). This inflammatory cascade peaks and subsides within 4-6 h of LPS injection. Rodents injected with LPS are perceptually discriminable to conspecifics from their saline-injected counterparts, as evidenced by increased avoidance behavior (Renault et al. 2008; Arakawa et al. 2009). Furthermore, smelling only the soiled bedding of LPS- versus saline-injected conspecifics evoked a similar heightened avoidance response, indicating that detectible odor cues of acute inflammation are present in the urine volatile profile (Arakawa et al. 2009, 2010; Boillat et al. 2015). Further support for the causal role of innate immune activation in the generation of this aversive odor cue comes from data demonstrating that infusion of pro-inflammatory IL-1 $\beta$ was sufficient to induce this heightened avoidance response and that infusion of anti-inflammatory IL-10 was sufficient to suppress it (Arakawa et al. 2010). Together, these studies demonstrate that nonhuman animals are capable of detecting cues of inflammation-not just infection-from the urine odors of conspecifics.

Evidence for a human capacity to communicate and detect conspecific sickness cues comes to a large extent from observational research and anecdotal reports that infectious and metabolic diseases emit disease-specific odor profiles (Penn and Potts 1998; Shirasu and Touhara 2011). However, these reports do not exemplify the inflammation detection necessary for successful behavioral avoidance of infected conspecifics but rather detection of more advanced disease states. Moreover, these data are often confounded by selection biases involving diet, lifestyle, and medication factors that may also affect endogenous odor (Parma et al. 2017). To bypass such confounds, we previously used an experimental LPS model of acute inflammation in otherwise healthy individuals to demonstrate that humanslike other mammals-are able to detect cues of acute inflammation. Participants found the axillary odors of human conspecifics collected during LPS-induced inflammation to be significantly more unpleasant, intense, and unhealthy than those collected from the same individuals after injection with a saline placebo (Olsson et al. 2014). Furthermore, we recently demonstrated that such axillary olfactory sickness cues were processed by a cortical network including primary and secondary olfactory areas in the brain and tended to reduce the likability of simultaneously presented faces (Regenbogen et al. 2017). Thus, axillary odor cues can provide information on the inflammatory status in humans, but whether similarly detectable cues exist in human urine odor is not yet known.

Studies of nonhuman animals have assessed the changes in endogenous odor caused by acute inflammation and the behavioral effects they induce in conspecifics using, with few exceptions, urine-a volatile source seldom studied in humans. The numerous anatomical and cultural adaptations that separate modern Homo sapiens from primates and even from earlier members of our own species have limited the contexts in which (and diminished the frequency with which) humans encounter conspecifics' urine. However, although the value to modern humans of any cue in conspecific urine odor may be limited, the presence or absence of a detectable cue with the potential to mediate avoidance is an independent question. Knowledge of the selection pressures favoring disease avoidance, the demonstrated ability of nonhuman animals to detect inflammation in urine volatiles, and the relatively recent shared ancestry of these animals and humans, in parallel with knowledge of the demonstrated human ability to detect inflammation in conspecific axillary volatiles and of the fact that the profile of volatile compounds in human urine overlaps significantly with that emitted by the human axillae (Penn et al. 2007), make human urine a promising potential source for cues of inflammation.

The aims of this study were 2-fold: first, we sought to determine whether humans can detect an acute inflammatory response in urine volatiles; second, we sought to identify potential volatile markers of the acute inflammatory response. Donors were injected with either LPS or a saline placebo, and urine was collected both before and after injection. To facilitate a double-blind design, we opted to use a low dose of LPS to induce a systemic increase in pro-inflammatory cytokines while minimizing overt behavioral and physical symptoms of sickness. The odor of urine samples was assessed by healthy human participants to identify perceptual differences induced by LPS treatment. Samples were also analyzed chemically by gas chromatography-mass spectrometry (GC-MS) to identify changes in volatile metabolites with the potential to discriminate LPS from Placebo treatments. We hypothesized that the changes to urine odor and volatile profile induced by LPS and Placebo treatment would be discriminable by perceptual and chemical assays, respectively, thereby indicating that human urine contains detectable cues of acute inflammation with the potential to mediate behavioral avoidance.

\section{Materials and methods}

\section{Endotoxin treatment and sample collection (urine and plasma) from donors}

Thirty-three healthy volunteers participated as urine donors after providing informed written consent, 20 in the LPS condition (13 women) and 13 in the Placebo condition (8 women). Donors were right-handed, medication-free, nonsmokers without any history of drug abuse, chronic pain, or psychiatric disorders. All women were premenopausal, and usage of hormonal contraceptives was allowed. Beginning $12 \mathrm{~h}$ prior to the first sample collection, donors followed a restricted diet free of food known to scent urine. Treatment and sample collection (urine and plasma) were performed using a randomized, double-blind, between-subjects design under the supervision of an attending physician in a hospital environment (Karshikoff et al. 2015). An injection of either $0.6 \mathrm{ng} \mathrm{LPS} / \mathrm{kg}$ body weight (Escherichia coli, Lot nr. G3E0609; United States Pharmacopeia) or phosphate-buffered saline solution was delivered intravenously 
at approximately 10:00 AM. Selection of a low LPS dosage facilitated maintenance of the double-blind design for the duration of the subsequent sample collection period. Urine samples were collected from each donor approximately $1 \mathrm{~h}$ pre- and $4 \mathrm{~h}$ postinjection in plastic cups and then immediately transferred to glass storage vials. The time elapsed between pre- (Pre) and postinjection urine sample (Post) collections did not differ significantly between conditions, $t(23.49)=0.42, P=0.677$ (LPS: $4.5 \pm 0.8$ h; Placebo: $4.4 \pm 0.8 \mathrm{~h}$ ). Plasma samples were collected from each donor preinjection (baseline, $0 \mathrm{~h}$ ) and at $1.5,3.5$, and $5 \mathrm{~h}$ postinjection in $10 \mathrm{~mL}$ ethylenediaminetetraacetic acid (EDTA) plasma tubes (BD Vacutainer) and were cold centrifuged at $1500 \times \mathrm{g}$ for $20 \mathrm{~min}$. Urine and plasma samples were frozen at $-70{ }^{\circ} \mathrm{C}$ on location immediately after collection and thawed $1 \mathrm{~h}$ before usage in either the chemical or behavioral assay. Urine samples were kept in freezers at $-70{ }^{\circ} \mathrm{C}$ for the entirety of the 2.25-2.75 year storage period between collection and analysis, except for the duration of express shipment to the United States, during which they were kept frozen on dry ice. All aspects of the treatment and sample collection portions of the experiment were approved by the Regional Ethical Review Board and performed at the Karolinska University Hospital (Solna, Sweden) in accordance with the Declaration of Helsinki on biomedical studies involving human subjects.

\section{Measures of an inflammatory response in donors}

Plasma samples were analyzed using the MILLIPLEX MAP high-sensitivity human cytokine kit (Millipore Corporation) with Luminex xMAP methodology (Luminex Corporation). Levels of TNF- $\alpha$, IL-6, IL-8, and IL-10 were measured at each collection time, and the area under the curve (AUC) was calculated for each cytokine to verify the presence and absence of an inflammatory response in LPS and Placebo donors, respectively.

\section{Sensory assessment participants}

Twenty-one participants (13 women, $26.2 \pm 3.6$ years, range: 19-32 years) performed the sensory assessment portion of the experiment after providing informed written consent. Participants were medication-free nonsmokers without any history of consciousness loss resulting from head trauma or hormonal, autoimmune, neurological, or psychiatric disorders-all factors with the potential to affect olfactory processing (Boesveldt et al. 2011). To verify that no participant had functional anosmia, a 6-item, 4-alternative, forced-choice odor identification test, selected from the original 40-item Monell Extended Sniffin' Sticks Identification Test (MONEX-40) (Freiherr et al. 2012), was administered. All participants correctly identified 4 or more odors, corresponding to a binomial probability significantly better than chance $(P<0.05)$. On the day of testing, participants used no scented body products and refrained from chewing gum and eating or drinking anything but water for the hour immediately prior to testing. All aspects of the sensory assessment portion of the experiment were approved by the institutional review board of the University of Pennsylvania and performed at the Monell Chemical Senses Center (Philadelphia, United States) in accordance with the Declaration of Helsinki on biomedical studies involving human subjects.

\section{Sensory assessment of donor urine samples}

Sensory testing was performed in rooms engineered for odor testing, equipped with a high air exchange rate to prevent lingering odors and red fluorescent lighting to prevent potential differences in stimulus color from influencing sensory assessments. Urine from 27 donors,
15 LPS (11 women, $26.5 \pm 6.0$ years, range: $20.6-41.0$ years) and 12 Placebo ( 7 women, $27.6 \pm 4.9$ years, range: $20.5-35.2$ years), was used in the sensory assessment portion of the experiment as, due to suspicion of contamination (e.g., noncompliance with food and hygiene instructions), urine from 6 donors was excluded. Donor age did not differ significantly between conditions $[t(24.97)=0.51, P=0.612]$. (Routine outlier identification in the GC-MS data later indicated that there were no outliers among the urine samples from 4 of the 6 excluded donors, and these were subsequently included in the chemometric analyses.) For sensory assessment, urine samples from LPS and Placebo donors were divided into multiple stimulus "triplets," each comprising 1 Post and 2 identical Pre volumes of $1.0 \mathrm{~mL}$ urine in $20 \mathrm{~mL}$ glass vials. Urine samples were vortexed before aliquoting to prevent any effects of sample fractionation. Each participant performed the same perceptual ratings and discrimination tasks on 3 LPS triplets ( 9 vials) and 3 Placebo triplets ( 9 vials) in 2 counterbalanced blocks of the testing session. In other words, each participant smelled Pre and Post urine samples from 3 LPS and 3 Placebo donors of the same sex. Stimulus triplets were pseudorandomly assigned to a participant and were never reused for any other participant.

In the 3-alternative, forced-choice discrimination tasks, participants were instructed to, on each of 11 trials, choose the odd one out of the 3 vials of a donor triplet, 1 vial with a donor's Post urine sample (target) and 2 with the same donor's Pre urine samples (lures). Trial order was pseudorandomized among the 3 donor triplets, either LPS or Placebo, depending on the experimental block, such that 2 of the 3 triplets were used on 4 trials, each, and 1 of the 3 triplets was used on only 3 of the 11 trials. Using intra-donor triplets precluded the possibility of discrimination based on donor identity, at which humans are adept (Lundström et al. 2008, 2009), rather than treatment, thereby preventing a sizeable confound.

Perceptual ratings of odor pleasantness, intensity, disgustingness, and donor sickness were performed on visual analog scales (VAS). Perceived odor pleasantness is widely believed to be the primary dimension of olfactory perceptual space and may be the basis of olfactory functioning (Yeshurun and Sobel 2010). Perceived intensity was chosen to assess the contribution of a quantitative, as opposed to qualitative, treatment-induced change in urine odor, and perceived disgust was selected on the basis of its hypothesized evolution as a disease-avoidance mechanism (Oaten et al. 2009). Duplicate ratings were collected in 2 rounds, each with a unique randomized order of vials from the 3 stimulus triplets of that block. Rating scales for perceived intensity, disgustingness, and sickness ranged from 0 to 10 , and the minima and maxima were defined as "Not at all" and "Most imaginable," respectively. The scale for perceived pleasantness ratings ranged from -5 to 5 , defined as "Most unpleasant imaginable" and "Most pleasant imaginable," respectively, and it contained a midpoint defined as "Neutral."

\section{Chemometric analyses of donor urine samples}

Headspace GC-MS and subsequent chromatographic data analyses assessed the potential differences in the profiles of volatile (i.e., odorous) organic compounds of urine from 33 LPS and Placebo donors. Triplicate $25 \mu \mathrm{L}$ urine aliquots from each donor were transferred to individual $20 \mathrm{~mL}$ glass sample vials and fortified with $10 \mu \mathrm{L}$ of an aqueous L-carvone solution so that $80.2 \mathrm{ng}$ was precisely delivered to each sample. Control samples consisted of "blank" vials, all of which lacked urine and contained only the aqueous Lcarvone solution. All samples were subjected to headspace analysis using a HT3 dynamic headspace analyzer (Teledyne Tekmar) outfitted with Supelco Trap K Vocarb 3000 thermal desorption trap 
(Sigma-Aldrich). Each sample vial was maintained at $40{ }^{\circ} \mathrm{C}$ and swept with helium for $45 \mathrm{~min}$ (flow rate of $75 \mathrm{~mL} / \mathrm{min}$ ), and the volatiles were collected on the trap. Trap contents were desorbed at $265^{\circ} \mathrm{C}$ directly into an ISQ single quadrupole GC-MS (Thermo Scientific) equipped with a $30 \mathrm{~m} \times 0.25 \mathrm{~mm}$ I.D. Stabilwax-DA fused-silica capillary column (Restek). The GC oven program had an initial temperature of $40{ }^{\circ} \mathrm{C}$ (held for $3.0 \mathrm{~min}$ ) followed by a ramp of $7.0{ }^{\circ} \mathrm{C} / \mathrm{min}$ to a final temperature of $230{ }^{\circ} \mathrm{C}$ (held for $6.0 \mathrm{~min}$ ). The mass spectrometer was used in scan mode from 33 to $400 \mathrm{~m} / \mathrm{z}$.

Chromatographic data were converted to NetCDF format for baseline correction, noise elimination, and peak alignment using MetAlign (Lommen 2009). Resulting multivariate data (consisting of all mass spectrometric responses exceeding a predefined threshold at each scan event) were further processed with the MSClust tool for mass spectra extraction and generation of individual selected ion chromatogram peak responses (Tikunov et al. 2012). Peak responses were scaled to the response of L-carvone, the internal standard. The resulting data set, arising from 201 urine sample analyses, consisted of a single scaled response per chromatographic peak identified in the headspace, which were submitted to statistical analyses.

\section{Statistical analyses and data handling}

Analyses were performed in R 3.0.2 (R Development Core Team 2013) using the lme4 package (Bates et al. 2015) for LMEM and multcomp package (Hothorn et al. 2008) for subsequent post hoc $t$-tests. $P$ values below 0.05 were considered statistically significant.

\section{Cytokine data}

Successful activation of the immune system by LPS injection in urine donors was assessed using AUC measures. Between-treatment (LPS and Placebo) comparisons were carried out for each cytokine with 2 -sided Welch's $t$-tests. These analyses were conducted on the larger group of donors included in chromatographic analyses $(n=31)$. Due to technical problems, plasma samples from 1 donor were missing ( $n=30 ; 18$ LPS, 12 Placebo).

\section{Perceptual discrimination data}

Discriminability of Pre and Post urine was compared between LPS and Placebo treatments using a paired $t$-test of mean number of correct trials.

\section{Perceptual ratings data}

To assess the effects of treatment on perceived dimensions of urine odor, linear mixed-effects models (LMEM) were constructed with Treatment (LPS and Placebo) and Time (Pre and Post) as fixed effects and Participant and Donor identity as random effects, which accounted for inter-participant differences in rating behavior and inter-donor differences in endogenous odor. One participant who misunderstood the rating procedure for perceived disgust was excluded from that model. All perceptual VAS ratings were natural log-transformed before analysis, and statistical results are reported in linear terms.

\section{Chromatographic data}

All carvone-scaled peak responses were subjected to principal components analysis using Unscrambler (CAMO Software) to visually identify outlier samples exhibiting undue influence or leverage in residual plots. Nine of the 201 samples were identified as outliers, and removal of these resulted in an absence of any posttreatment samples for 2 donors, thereby effectively excluding those donors from further analysis. Samples from 31 donors were retained-19 donors from the LPS condition (13 women, $26.1 \pm 5.6$ years, range: $20.1-$ 41.1 years) and 12 donors from the Placebo condition ( 7 women, $27.6 \pm 4.9$ years, range: $20.5-35.2$ years). Donor age did not differ significantly between conditions $[t(25.87)=0.77, P=0.446]$. Among retained sample data, means from repeated chemical analyses of the same urine sample were calculated for each carvone-scaled peak response. Treatment differences were calculated for each donor by subtracting mean Pre responses from mean Post responses. To reduce the number of variables for analysis, the VARCLUS procedure in SAS was used to group the 42 difference responses into related clusters. Component scores were then calculated from VARCLUS scoring coefficients and standardized differences using the SCORE procedure (SAS). Seven component scores were subjected to multivariate analysis of variance with univariate ANOVA to determine which clusters were impacted by treatment (the lone between-subjects effect). The individual chemical variables contributing to significant clusters were identified. Standardized mean peak responses for these individual chemical variables were submitted to LMEM analyses constructed with Treatment (LPS and Placebo) and Time (Pre and Post) as fixed effects and Donor identity as a random effect.

\section{Results}

\section{Verification of an inflammatory response in donors}

Successful triggering of the innate immune system was demonstrated by significant LPS-induced increases in AUC measures of plasma cytokine concentrations, relative to Placebo: TNF- $\alpha$, $t(17.122)=4.21, P=0.0006 ;$ IL-6, $t(17.184)=4.393, P=0.0004$; IL- $8, t(17.043)=3.697, P=0.0018$; and IL-10, $t(17.319)=5.24$, $P<0.0001$ (Figure 1).

\section{Perceptual discrimination}

Results from the discrimination tasks (Figure 2) indicated that the perceptual discriminability differed significantly between treatments [LPS $=4.24$ correct trials \pm standard deviation $1.87,38.5 \pm 17.0 \%$; Placebo $=5.71 \pm 1.76,51.9 \pm 16.0 \% ; t(20)=2.62, P=0.016]$, with participants correctly discriminating Post from Pre in more Placebo than LPS trials.

\section{Perceptual ratings}

A significant interaction of Treatment (LPS and Placebo) and Time (Pre and Post) on perceived urine odor intensity, $\chi^{2}(1)=5.64$, $P=0.018$, was mirrored by a statistical trend for perceived donor sickness, $\chi^{2}(1)=3.33, P=0.068$, but not for odor disgustingness, $\chi^{2}(1)=2.61, P=0.106$, and pleasantness, $\chi^{2}(1)=0.19, P=0.666$ (Figure 3). The nominal pattern of results suggests that the natural decrease in urine odor averseness observed in Placebo donors over the day was disrupted by LPS treatment, rendering postinjection LPS samples more aversive than their Placebo counterparts.

As the correlations between the 4 types of ratings were high (absolute values between 0.52 and 0.80 ), a factor analysis was conducted, indicating a single factor solution (absolute factor loadings between 0.69 and 0.92 ). A factor score-indicating levels of intensity, pleasantness, disgust, and donor sickness-was calculated for each trial. Another analysis, like that described earlier, revealed a significant interaction of Treatment and Time on the factor score, $\chi^{2}(1)=4.36$, $P=0.037$. In accordance with the results above, the factor score tended to decrease from the Pre- to the Post-measurement, but less so for LPS-treated participants (Figure 4). 


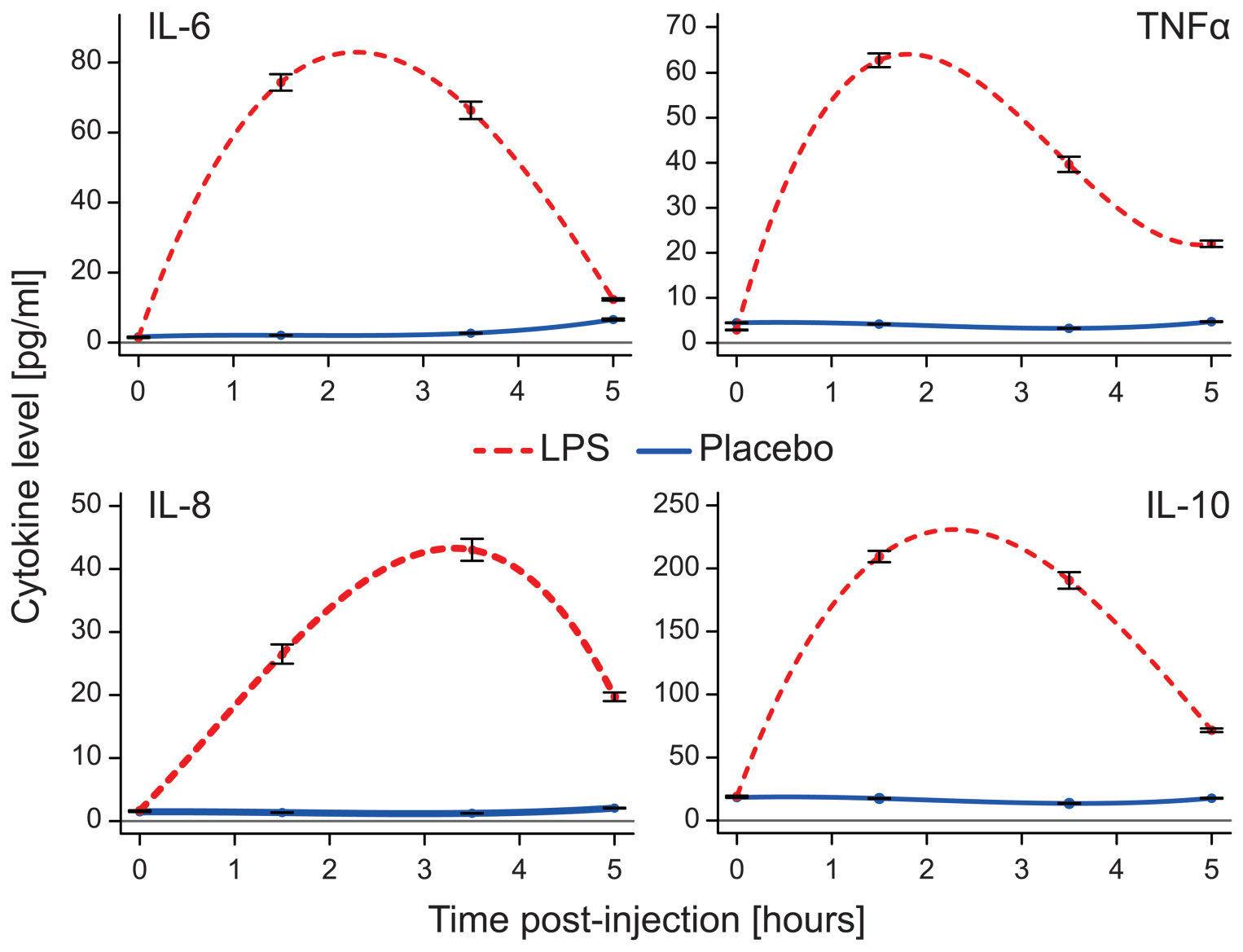

Figure 1. Levels of cytokines (pg/ml). Error bars denote standard error of the mean.

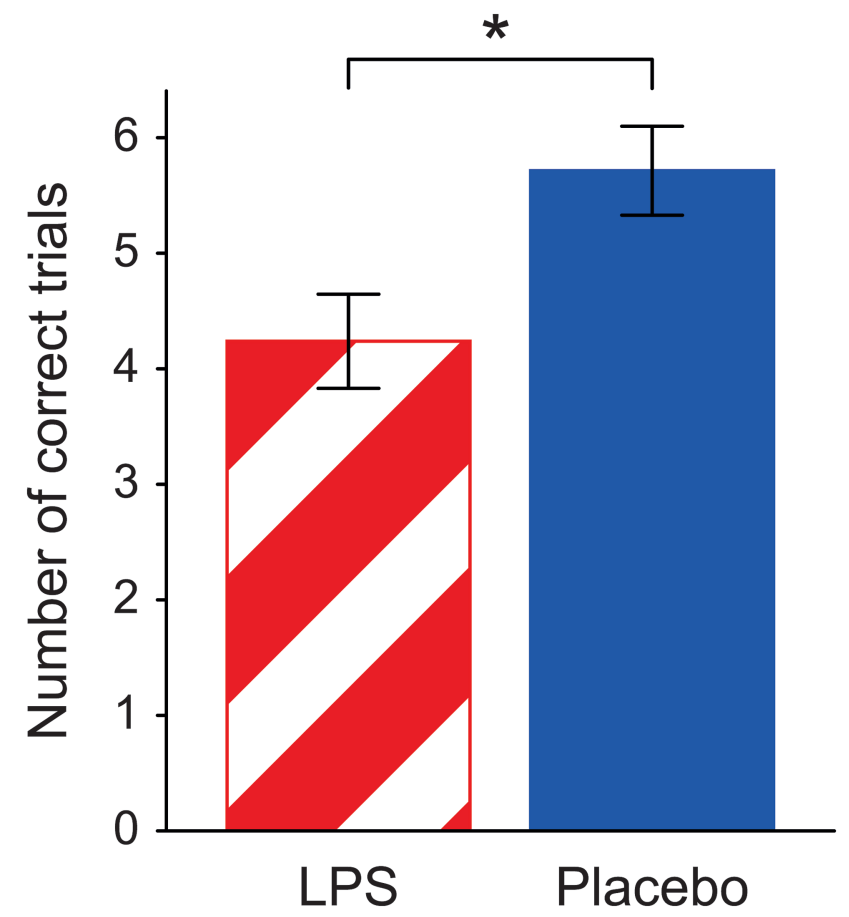

Figure 2. Number of correct discriminations of Pre and Post urine for LPS and Placebo samples. Error bars denote standard error of the mean, and the asterisk indicates significance $\left({ }^{*} P<0.05\right)$.

\section{Chemometric analysis}

Urine samples were submitted to chemical analysis and subsequent chromatographic data processing to assess the pattern of treatment effects on the volatile profile. Cluster analysis revealed 7 clusters, ranging from 22 compounds to 2 compounds. Analysis of cluster scores indicated that there was a significant treatment effect for only 1 of the 7 clusters $(P=0.015)$. The 3 odorants comprising this cluster were chromatographic peaks identified by comparison to known standards as: acetophenone, pyrrole, and benzaldehyde. Analysis with LMEM revealed significant or statistical trends for the interaction of Treatment (LPS and Placebo) and Time (Pre and Post) for acetophenone, $\chi^{2}(1)=10.79, P=0.001 ;$ pyrrole, $\chi^{2}(1)=8.75, P=0.003$; and benzaldehyde, $\chi^{2}(1)=3.08, P=0.079$. In short, pyrrole and acetophenone were significantly more abundant following LPS treatment, as indicated by Figure 5 .

\section{Discussion}

The results presented here suggest that within hours of exposure to even a relatively weak immune challenge, acute inflammation can induce changes to human urine odor that are detectable by both human and chemical assays.

More specifically, the results revealed a natural rhythm-a pattern over time - of decreasing urine odor averseness through the day (from morning [Pre] to midday [Post] collections), a change that is disrupted by upregulation of the innate immune system. The time 
course of this natural rhythm, observed in Placebo urine, is unsurprising given the well-established facts that the kidneys' glomerular filtration rate decreases overnight and increases during the day (Koopman et al. 1989) and that a significant proportion of the human urine metabolome exhibits a diurnal rhythm (Giskeødegård et al. 2015)
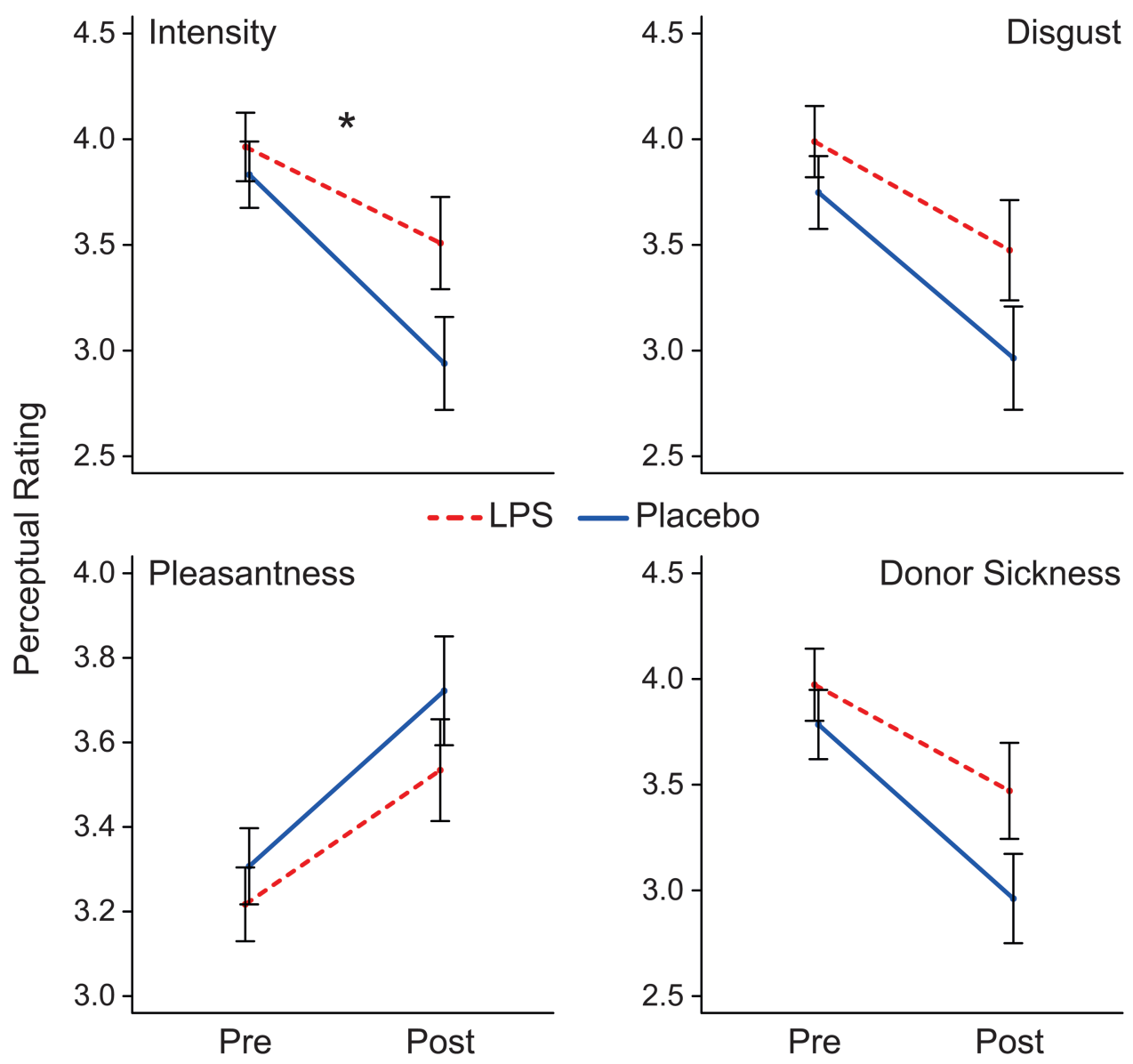

Figure 3. Perceptual ratings of Pre and Post urine for LPS and Placebo samples. Error bars denote standard error of the mean, the asterisk indicates significance $\left({ }^{*} P<0.05\right)$. The scales for ratings range from 0 "Not at all" to 10 "Most imaginable," with the exception of the scale for pleasantness, which ranges from -5 "Most unpleasant imaginable" to 5 "Most pleasant imaginable."

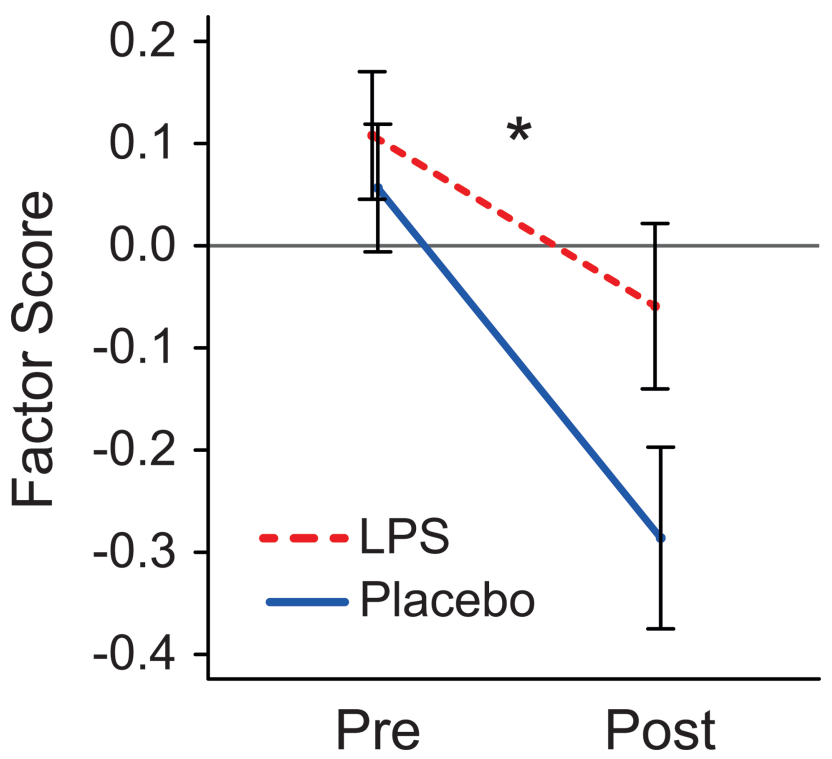

Figure 4. The factor score-reflecting a hypothesized latent variable for levels of intensity, pleasantness, disgust, and donor sickness-for Pre and Post urine for LPS and Placebo samples. Error bars denote standard error of the mean, and the asterisk indicates significance $\left({ }^{*} P<0.05\right)$. 

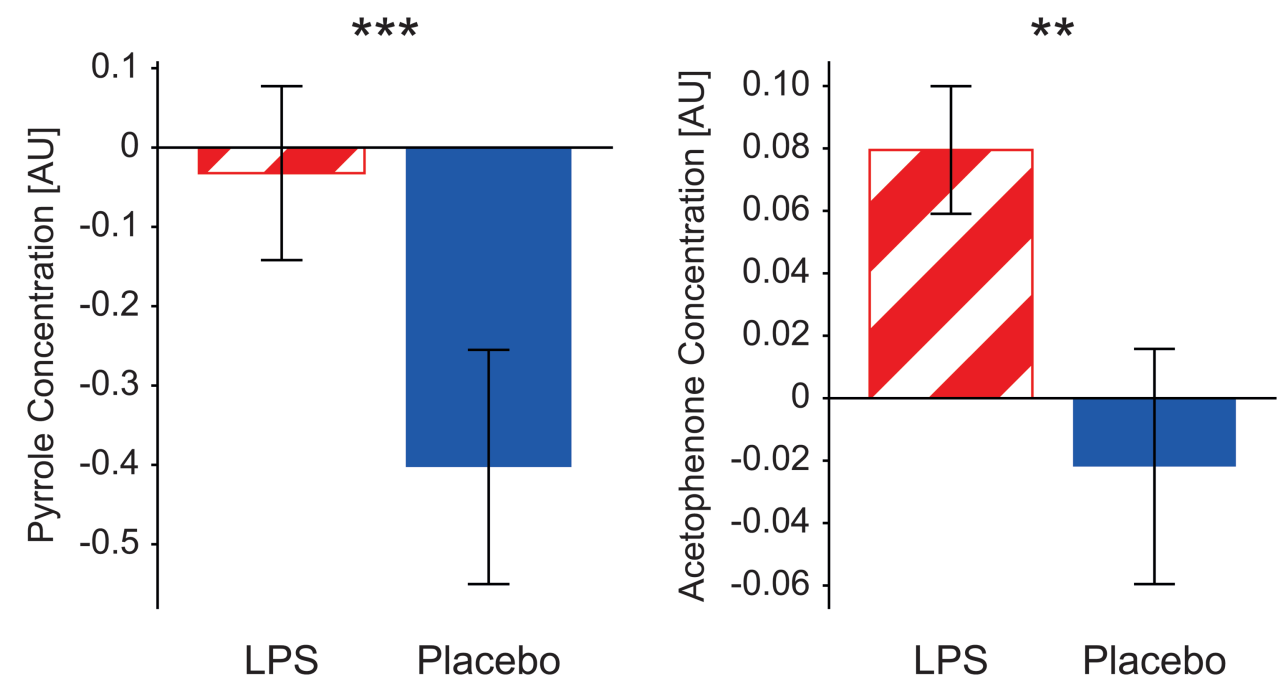

Figure 5. Differences between Pre- and Post-measurements of carvone-normalized pyrrole concentrations and acetophenone concentrations in LPS- and Placebo-treated urine. Error bars denote standard error of the mean, AU refers to arbitrary units of differences, and asterisks indicate significance ${ }^{*} P<0.05$, $\left.{ }^{*} P<0.01,{ }^{* * *} P<0.001\right)$.

Although it remains to be conclusively demonstrated what mediated the difference in discriminability of Pre and Post urine between the healthy, Placebo and LPS treatments, our chemometric data and perceptual ratings offer enticing clues. In the absence of inflammation, the chemometric data revealed a natural decrease in pyrrole abundance from Pre to Post collection times-a change sufficient for linear discriminant models to differentiate Post from Pre Placebo urine. This decrease in pyrrole abundance was absent under LPS treatment. The perceptual ratings may reflect this natural drop of pyrrole abundance in the decrease of perceived intensity observed posttreatment for Placebo, but not LPS, samples (Figure 3). This suggests that pyrrole may be a urinary volatile with a diurnal rhythm that is interrupted by activation of the innate immune system, resulting in higher-than-normal pyrrole levels in Post LPS urine samples. LPS did induce a significant change in the urinary volatile profile in the form of increased acetophenone abundance. No such change in acetophenone abundance was seen in Placebo samples, suggesting that this increase is because of treatment alone rather than to any natural rhythmic changes.

By what mechanism(s) inflammation alters endogenous odor volatiles is not well known (Arakawa et al. 2011), especially not for the time scale investigated here (hours). The innate immune system, which is reliably activated by the canonical stimulus LPS, has repeatedly been linked to endogenous odors both in animals (Beauchamp et al. 1985; Beauchamp and Yamazaki 2005; Arakawa et al. 2010) and in humans (Olsson et al. 2014), but whether the effects demonstrated in this study are directly mediated by inflammatory processes cannot be conclusively demonstrated. However, the 2 odorous volatiles, pyrrole and acetophenone, that were significantly elevated as a function of LPS have previously been suggested as biomarkers for various diseases and pathogen infections. The metabolite pyrrole has been identified as elevated in the metabolic disorder phenylketonuria disorder (Burke et al. 1983). Increases in urine concentrations of acetophenone have previously been indicated in the mouse models of lung cancer (Matsumura et al. 2010). How elevated levels of pyrrole and acetophenone and activation of the immune system are linked is currently not clear. However, based on their known involvement in different pathologies, it is conceivable that these 2 odorous volatiles together enabled discrimination between treatments in the cluster model, not as disease- or pathogen-specific cues but rather as indicators of the general inflammatory response.

An understanding of the perception of health—or sickness-as a complex process of integrating multiple sensory cues, often from multiple sensory modalities, is important for appreciating the role that even the smallest cues can play in contributing to the differentiation of sick and healthy conspecifics. We have demonstrated an additive effect of olfactory-visual integration in the perception of sickness cues from facial photographs and body odors (Regenbogen et al. 2017). And we recently demonstrated (Axelsson et al. 2018) that health perception is influenced by a number of subtle visual cues of inflammation-such as paleness of the skin and lips, redness of the eyes, and droopiness of the eyelids. With knowledge of the role that sensory integration plays in health perception, it becomes easier to appreciate the potency of disease detection-even during the early stages of inflammation when cues may be weak.

These results, akin to animal findings, indicate that there is an olfactory cue of inflammation in human urine detectable to conspecifics. The perceived differences of urine odor intensity, sickness, and disgustingness indicate that we find the urine of other humans hours after onset of an inflammatory response more aversive than the urine of healthy counterparts at the same time of day, when baseline (Pre) measures are corrected for. The relative differences in abundances of pyrrole and acetophenone, and in discriminability between Placebo and LPS urine, offer tantalizing glimpses into, respectively, the potential chemical mechanisms that may underpin and the potential behavioral consequences that may rest on these perceptual differences. However, these data do not provide causal links between the chemical changes, perceived changes, and discriminability changes.

Future studies, based on these results, can bridge these knowledge gaps and refine our description of this inflammation cue in human urine by optimizing and narrowing the scope of their experimental designs. The putative volatile mediators identified here, pyrrole and acetophenone, can be targeted directly in follow-up studies, thereby avoiding data reduction practices. To help mitigate the potential for urine concentration to bias between-treatment perceptual or chemometric measures, future studies should standardize hydration levels among their urine donors. These data, however, appear unaffected by this confound, 
as the chemometric analyses failed to demonstrate any general shift in abundancy as a function of LPS treatment. Limiting the effects of urine sample preservation by minimizing the handling of samples between collection and analysis would also strengthen the results of future studies.

The results of this study strengthen the rising view that systemic inflammation entails physiological changes that are perceptually detectable to other humans, possibly serving adaptive purposes during evolution as a behavioral defense against infectious disease, a central threat to survival.

\section{Funding}

This work was supported by the Knut and Alice Wallenberg Foundation [KAW 2012.0141 to J.N.L.]; the Swedish Research Council [421-2012-1125 and 2016-02742 to M.J.O.; 2014-1346 to J.N.L.]; and the Swedish Foundation for Humanities and Social Sciences [P12-1017 to M.J.O.].

\section{Acknowledgements}

The authors wish to thank Ms Kelly Dougherty and Mr Jonathan Namini for help with data collection.

\section{Conflicts of interest}

None.

\section{References}

Arakawa H, Arakawa K, Deak T. 2009. Acute illness induces the release of aversive odor cues from adult, but not prepubertal, male rats and suppresses social investigation by conspecifics. Behav Neurosci. 123:964-978.

Arakawa H, Arakawa K, Deak T. 2010. Sickness-related odor communication signals as determinants of social behavior in rat: a role for inflammatory processes. Horm Behav. 57:330-341

Arakawa H, Cruz S, Deak T. 2011. From models to mechanisms: odorant communication as a key determinant of social behavior in rodents during illness-associated states. Neurosci Biobehav Rev. 35:1916-1928.

Axelsson J, Sundelin T, Olsson MJ, Sorjonen K, Axelsson C, Lasselin J, Lekander M. 2018. Identification of acutely sick people and facial cues of sickness. Proc Biol Sci. 285. doi: 10.1098/rspb.2017.2430

Bates D, Mächler M, Bolker B, Walker S. 2015. Fitting linear mixed-effects models using lme4. J Stat Software. 67:1-48.

Beauchamp GK, Yamazaki K. 2005. Individual differences and the chemical senses. Chem Senses. 30(1 Suppl):i6-i9.

Beauchamp GK, Yamazaki K, Wysocki CJ, Slotnick BM, Thomas L, Boyse EA. 1985. Chemosensory recognition of mouse major histocompatibility types by another species. Proc Natl Acad Sci U S A. 82:4186-4188.

Beutler BA. 2009. TLRs and innate immunity. Blood. 113:1399-1407.

Boesveldt S, Lindau ST, McClintock MK, Hummel T, Lundstrom JN. 2011. Gustatory and olfactory dysfunction in older adults: a national probability study. Rhinology. 49:324-330.

Boillat M, Challet L, Rossier D, Kan C, Carleton A, Rodriguez I. 2015. The vomeronasal system mediates sick conspecific avoidance. Curr Biol. 25:251-255.

Burke DG, Halpern B, Malegan D, McCairns E, Danks D, Schlesinger P, Wilken B. 1983. Profiles of urinary volatiles from metabolic disorders characterized by unusual odors. Clin Chem. 29:1834-1838.

Curtis VA. 2014. Infection-avoidance behaviour in humans and other animals. Trends Immunol. 35:457-464.

Dantzer R, O'Connor JC, Freund GG, Johnson RW, Kelley KW. 2008. From inflammation to sickness and depression: when the immune system subjugates the brain. Nat Rev Neurosci. 9:46-56 de Groot JHB, Smeets MAM, Kaldewaij A, Duijndam MJA, Semin GR. 2012. Chemosignals communicate human emotions. Psychol Sci. 23:1417-1424.

Eggert F, Luszyk D, Haberkorn K, Wobst B, Vostrowsky O, Westphal E, Bestmann HJ, Müller-Ruchholtz W, Ferstl R. 1998. The major histocompatibility complex and the chemosensory signalling of individuality in humans. Genetica. 104:265-273.

Ehman KD, Scott ME. 2001. Urinary odour preferences of MHC congenic female mice, Mus domesticus: implications for kin recognition and detection of parasitized males. Animal Behaviour. 62:781-789.

Ehman KD, Scott ME. 2002. Female mice mate preferentially with non-parasitized males. Parasitology. 125:461-466.

Freiherr J, Gordon AR, Alden EC, Ponting AL, Hernandez MF, Boesveldt S, Lundström JN. 2012. The 40-item Monell Extended Sniffin' Sticks Identification Test (MONEX-40). J Neurosci Methods. 205:10-16.

Fumagalli M, Sironi M, Pozzoli U, Ferrer-Admetlla A, Pattini L, Nielsen R. 2011. Signatures of environmental genetic adaptation pinpoint pathogens as the main selective pressure through human evolution. PLoS Genet. 7:e1002355.

Giskeødegård GF, Davies SK, Revell VL, Keun H, Skene DJ. 2015. Diurnal rhythms in the human urine metabolome during sleep and total sleep deprivation. Sci Rep. 5:14843.

Hothorn T, Bretz F, Westfall P. 2008. Simultaneous inference in general parametric models. Biom J. 50:346-363.

Jones DS, Podolsky SH, Greene JA. 2012. The burden of disease and the changing task of medicine. N Engl J Med. 366:2333-2338.

Karshikoff B. 2015. Sickness behavior: immune system influences on brain and behavior [PhD]. [Stockholm (Sweden)]: Karolinska Institutet.

Karshikoff B, Lekander M, Soop A, Lindstedt F, Ingvar M, Kosek E, Olgart Höglund C, Axelsson J. 2015. Modality and sex differences in pain sensitivity during human endotoxemia. Brain Behav Immun. 46:35-43.

Kavaliers M, Colwell DD. 1995a. Discrimination by female mice between the odours of parasitized and non-parasitized males. Proc Biol Sci. 261:31-35.

Kavaliers M, Colwell DD. 1995b. Odours of parasitized males induce aversive responses in female mice. Anim Behav. 50:1161-1169.

Kiesecker JM, Skelly DK, Beard KH, Preisser E. 1999. Behavioral reduction of infection risk. Proc Natl Acad Sci U S A. 96:9165-9168.

Kimball BA, Opiekun M, Yamazaki K, Beauchamp GK. 2014. Immunization alters body odor. Physiol Behav. 128:80-85.

Koopman MG, Koomen GC, Krediet RT, de Moor EA, Hoek FJ, Arisz L. 1989. Circadian rhythm of glomerular filtration rate in normal individuals. Clin Sci (Lond). 77:105-111.

Lommen A. 2009. MetAlign: interface-driven, versatile metabolomics tool for hyphenated full-scan mass spectrometry data preprocessing. Anal Chem. 81:3079-3086.

Lundström JN, Boyle JA, Zatorre RJ, Jones-Gotman M. 2008. Functional neuronal processing of body odors differs from that of similar common odors. Cereb Cortex. 18:1466-1474.

Lundström JN, Boyle JA, Zatorre RJ, Jones-Gotman M. 2009. The neuronal substrates of human olfactory based kin recognition. Hum Brain Mapp. 30:2571-2580.

Matsumura K, Opiekun M, Oka H, Vachani A, Albelda SM, Yamazaki K, Beauchamp GK. 2010. Urinary volatile compounds as biomarkers for lung cancer: a proof of principle study using odor signatures in mouse models of lung cancer. PLoS One. 5:e8819.

Mitro S, Gordon AR, Olsson MJ, Lundström JN. 2012. The smell of age: perception and discrimination of body odors of different ages. PLoS One. 7:e38110.

Oaten M, Stevenson RJ, Case TI. 2009. Disgust as a disease-avoidance mechanism. Psychol Bull. 135:303-321.

Olsson MJ, Lundström JN, Kimball BA, Gordon AR, Karshikoff B, Hosseini N, Sorjonen K, Olgart Höglund C, Solares C, Soop A, et al. 2014. The scent of disease: human body odor contains an early chemosensory cue of sickness. Psychol Sci. 25:817-823.

Osada K, Yamazaki K, Curran M, Bard J, Smith BPC, Beauchamp GK. 2003. The scent of age. Proc Biol Sci. 270:929-933.

Parma V, Gordon AR, Cecchetto C, Cavazzana A, Lundström JN, Olsson MJ. 2017. Processing of human body odors. In Buettner A, editor, Springer handbook of odor. Cham (Switzerland): Springer International. p. 963-986. 
Penn DJ, Oberzaucher E, Grammer K, Fischer G, Soini HA, Wiesler D, Novotny MV, Dixon SJ, Xu Y, Brereton RG. 2007. Individual and gender fingerprints in human body odour. J R Soc Interface. 4:331-340.

Penn D, Potts WK. 1998. Chemical signals and parasite-mediated sexual selection. Trends Ecol Evol. 13:391-396.

R Development Core Team. 2013. R: a language and environment for statistical computing. (Version 3.0.2). Vienna (Austria): R Foundation for Statistical Computing. Retrieved from http://www.R-project.org/

Regenbogen C, Axelsson J, Lasselin J, Porada DK, Sundelin T, Peter MG, Lekander M, Lundström JN,Olsson MJ. 2017. Behavioral and neural correlates to multisensory detection of sick humans. Proc Natl Acad Sci U S A. 114:6400-6405.

Renault J, Gheusi G, Aubert A. 2008. Changes in social exploration of a lipopolysaccharides-treated conspecific in mice: role of environmental cues. Brain Behav Immun. 22:1201-1207.
Schaller M, Duncan LA. 2007. The behavioral immune system its evolution and social psychological implications. In: Forgas PJ, Haselton MG, von hippel W, editors. Evolution and the social mind: evolutionary psychology and social cognition. New York: Psychology Press. 293-307.

Shirasu M, Touhara K. 2011. The scent of disease: volatile organic compounds of the human body related to disease and disorder. $J$ Biochem. 150:257-266.

Tikunov YM, Laptenok S, Hall RD, Bovy A, de Vos RC. 2012. MSClust: a tool for unsupervised mass spectra extraction of chromatography-mass spectrometry ion-wise aligned data. Metabolomics. 8:714-718.

Wyatt TD. 2014. Pheromones and animal behavior: chemical signals and signatures. 2nd ed. Cambridge (UK): Cambridge University Press.

Yeshurun Y, Sobel N. 2010. An odor is not worth a thousand words: from multidimensional odors to unidimensional odor objects. Annu Rev Psychol. 61:219-41, C1. 